\title{
Factores personales y nivel de actividad física del adulto mayor de una casa de día de una comunidad rural
}

\author{
Personal factors and level of physical activity of the elderly in a house of day
}

Adriana Guerrero-García ${ }^{a}$,Gabriela Maldonado-Muñiz ${ }^{b}$,Claudia A. Trejo-García ${ }^{c}$

\begin{abstract}
:
Introduction: Older adults who engage in physical activity can benefit from it; as greater autonomy, satisfaction and excitement would highlight a state of well-being behaviour. Personal factors, according to Nola J. Pender's promotion model, predict a given behavior as physical activity. Objective: To determine the relationship between personal factors and the level of physical activity of older adults in a day-care center. Material and methods: quantitative, correlational, observational, and transversal study; universe of 60 people registered to the activities of the Mission Hidalgo day care center, over 65 years old, who accepted to participate; Rosenberg's selfesteem scale, a personal factors questionnaire, and the GPAQ were used to measure the level of physical activity, signed by informed consent. Results: Personal factors: Biological: 59.3\% women, mean age 75.78 \pm 7.79 years; Psychological: $44.7 \%$ showed high selfesteem, $42.6 \%$ medium and $16.7 \%$ low; Socio-cultural: $77.8 \%$ were Catholic; $83.3 \%$ received government support, $31.5 \%$ were widowed, $59.3 \%$ were in the home and $35.2 \%$ in the field, $59.3 \%$ with primary school education. Physical activity: $64.8 \%$ had a high level, $24.1 \%$ a medium level, and $11.1 \%$ a low level. Pearson correlated the level of physical activity with gender (biological factors) and religion (sociodemographic factors). Conclusions: In the population studied, a high level of physical activity predominates, influenced by personal factors such as age and religion. The self-esteem of the older adult is high, free union prevails, no studies, income is obtained from government support, these personal factors do not influence the physical activity of the older adult in a rural community.
\end{abstract}

\section{Keywords:}

Physical Activity, Older Adult, Personal Factors.

\begin{abstract}
Resumen:
Introducción: Los adultos mayores que realizan actividad física pueden beneficiarse de ella; como mayor autonomía, satisfacción y emoción lo que resaltaría un comportamiento de estado de bienestar. Los factores personales de acuerdo al modelo de promoción de Nola J. Pender, predicen un comportamiento determinado como a actividad física. Objetivo: Determinar la relación entre los factores personales y el nivel de actividad física de los adultos mayores de una casa de día. Material y métodos: estudio cuantitativo, correlacional, observacional, transversal; universo de 60 personas inscritas a las actividades de la casa de día de la Misión Hidalgo, mayores de 65 años, que aceptaron participar; se utilizó la escala de autoestima de Rosenberg, un cuestionario de factores personales y el GPAQ para medir el nivel de actividad física, se firmó de consentimiento informado. Resultados: Factores personales: bio lógicos: 59.3\% mujeres, edad media de $75.78 \pm 7.79$ años; Psicológicos: $44.7 \%$ evidenciaron autoestima elevada, $42.6 \%$ media y $16.7 \%$ baja; Socioculturales: $77.8 \%$ profesaban religión católica; $83.3 \%$ recibían apoyo de gobierno, $31.5 \%$ eran viudo, $59.3 \%$ se dedicaban al hogar y $35.2 \%$ al campo, $59.3 \%$ con escolaridad de primaria. Actividad física: $64.8 \%$ obtuvo nivel alto, $24.1 \%$ medio y $11.1 \%$ bajo. Se realizó correlación de Pearson encontrando significancia estadística del nivel de actividad física con género (factores biológicos) y religión (factores sociodemográficos). Conclusiones: En la población estudiada predomina un nivel alto de actividad física influyendo los factores personales como lo son la edad y la religión. La autoestima del adulto mayor es elevada, prevalece la unión libre, sin estudios, los ingresos son obtenidos por parte de apoyo gubernamental, estos factores personales no influyen en la actividad física del adulto mayor de una comunidad rural.
\end{abstract}

\section{Palabras Clave:}

Actividad Física, Adulto Mayor, Factores Personales

\footnotetext{
a Universidad Autónoma del Estado de Hidalgo, Escuela Superior de Tlahuelilpan, https://orcid.org/0000-0003-4006-8643, Email: gu355187@uaeh.edu.mx

b Autor de Correspondencia, Universidad Autónoma del Estado de Hidalgo, Escuela Superior de Tlahuelilpan, https://orcid.org/ https://orcid.org/0000-0002-4967-1812,Email: gmaldonado@uaeh.edu.mx
}

c Universidad Autónoma del Estado de Hidalgo, Escuela Superior de Tlahuelilpan, https://orcid.org/0000-0003-2205-7879, Email: ctrejo@uaeh.edu.mx 


\section{Introducción}

A nivel mundial, uno de cada cuatro adultos mayores no tiene un nivel suficiente de actividad física. Más del $80 \%$ de la población del mundo no tiene un nivel suficiente de actividad física. El 56\% de los Estados Miembros de la OMS ha puesto en marcha políticas para reducir la inactividad física. Los Estados Miembros de la OMS han acordado reducir la inactividad física en un 10\% para 2025. (1)

Debido al aumento de la esperanza de vida y a la disminución de la tasa de fecundidad, la proporción de personas mayores de 60 años está aumentando más rápidamente que cualquier otro grupo de edad en casi todos los países. El envejecimiento de la población puede considerarse un éxito de las políticas de salud pública y el desarrollo socioeconómico, pero también constituye un reto para la sociedad, que debe adaptarse a ello para mejorar al máximo la salud y la capacidad funcional de las personas mayores, así como su participación social y su seguridad ${ }^{(2)}$ su actividad diaria está vinculada a labores domésticas o al cuidado de niños y/o otros adultos; en el tiempo libre, las actividades son de carácter individual, sedentarias, realizadas en el marco del hogary con poca participación en ejercicios físicos sistemáticos, ${ }^{(3)}$ es una etapa que conlleva significados, estructuras, obligaciones y relaciones; se caracteriza por pérdidas de capacidades funcionales, de recursos, de oportunidades, de plenitud, ésta situación conlleva un grado de dependencia a la familia, a los servicios públicos de salud o programas de bienestar social. ${ }^{(4)}$

Se considera actividad física cualquier movimiento corporal producido por los músculos esqueléticos que exija gasto de energía. ${ }^{\left({ }^{5}\right)}$ Para los adultos de este grupo de edades, la actividad física consiste en actividades recreativas o de ocio, desplazamientos (por ejemplo, paseos caminando $o$ en bicicleta), actividades ocupacionales (cuando la persona todavía desempeña actividad laboral), tareas domésticas, juegos, deportes 0 ejercicios programados en el contexto de las actividades diarias, familiares y comunitarias. ${ }^{(6)}$

El modelo de promoción de la salud, integra las perspectivas de enfermería y las ciencias conductuales sobre los factores que influyen en la salud y motivan a los individuos a realizar comportamientos a la ampliación de la salud. ${ }^{(7)}$ Ver figura 1.

El objetivo de la presente investigación fue determinar los factores personales y la actividad física del adulto mayor de la casa de día del municipio de La Misión, Hidalgo.



Figura 1. Modelo de promoción de la salud de Nola J. Pender.

\section{Método}

Es un estudio de tipo cuantitativo, correlacional con diseño observacional, transversal; esta investigación se llevó a cabo en la casa de día de la Misión, Hidalgo, en enero 2020. Con un universo de 60 personas inscritos en la casa de día, mayores de 65 años, que aceptaron participar firmando el consentimiento informado.

Se solicitó autorización al personal mediante un oficio donde se estableció un horario y espacio en el cual se realizó la aplicación de los instrumentos y consentimiento informado.

Para la recolección de datos se utilizó la escala de autoestima de Rosenberg, la escala consta de 10 ítems, frases de las cinco están enunciadas de forma positiva y cinco de forma negativa para controlar el efecto de la aquiescencia administrada; la categorización de resultados son tres: elevada, media, baja; ${ }^{(8)}$ además de un cuestionario de factores personales y de GPAQ ${ }^{(9)}$ se utiliza para medir la actividad física; consta de 16 ítems para evaluar la actividad física desarrollada en tres dominios (actividadfísica ocupacional, relacionada con el transporte y actividad física en el tiempo libre) y abarca varios componentes de la actividad física, tales como intensidad, duración y frecuencia, teniendo como clasificación tres: nivel bajo, medio y alto.

\section{Resultados.}

La población de estudio fue de 54 adultos mayores de la casa de día de la Misión, Hidalgo, en la población se caracteriza que en género (factores personales biológicos) con mayor predisposición fue el femenino $59.3 \%$, mientras que el $40.7 \%$ representa en sexo masculino, en cuanto a la edad se encontró que la edad mínima fue de 61 años de edad y la máxima de 93 años

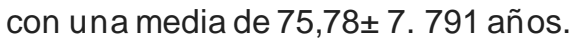

En cuanto a factores personales socioculturales en religión un $77.8 \%$ profesan la religión católica mientras el $22.2 \%$ son de la religión cristiana; en estado socio 
económico predomina $83.3 \%$ el apoyo de gobierno, el $11.1 \%$ apoyo familiar y $3.7 \%$ trabajo, $1.9 \%$ pensión, en estado civil se encontró que el $31.5 \%$ es viuda(o), el $27.8 \%$ casada(o) y el $25.9 \%$ unión libre, $11.1 \%$ soltero y $3.7 \%$ divorciada (o), en cuanto a la ocupación $59.3 \%$ es ama de casa, $35.2 \%$ trabaja en campo y un $5.6 \%$ tiene un trabajo laboral, respecto con la escolaridad encontramos un $59.3 \%$ primaria, el $25.9 \%$ sin estudios y $14.8 \%$ secundaria, en factores personales psicológicos $42.6 \%$ se encuentran en autoestima media, $40.7 \%$ en elevada y $16.7 \%$ en bajo.

En cuanto a la actividad física se encontró un $64.8 \%$ en un nivel alto, $24.1 \%$ en nivel medio y $11.1 \%$ en bajo.

Tabla 1. "Frecuencia y porcentaje del nivel de actividad física de los adultos mayores de la casa de día, La Misión, Hidalgo, México, 2019"

\begin{tabular}{|lcc|}
\hline $\begin{array}{l}\text { Nivel de Actividad } \\
\text { Física }\end{array}$ & f. & $\%$. \\
\hline Alto. & 35 & 64.8 \\
Medio. & 13 & 24.1 \\
Bajo. & 6 & 11.1 \\
\hline
\end{tabular}

Fuente: Cuestionario Mundial sobre Actividad Física (GPAQ) $\mathrm{n}=54$

Con la prueba de hipótesis de correlación de Pearson se pudo demostrar que los factores personales si influyen en la actividad física como lo son el género con una significación de 0,30 en género y 0,37 en religión siendo menor a 0.05 .

A lo que se puede decir que tiene un nivel de correlación positiva débil aprobando la hipótesis de trabajo $\mathrm{Hi}$ : los factores personales influyen en la actividad física del adulto mayor.

\section{Discusión.}

En este estudio se determinan los factores personales que influyen en la actividad física de adulto mayor en la casa de día, en La Misión Hidalgo. Utilizando la escala de autoestima de Rosemberg, cuestionario de factores personales y el instrumento de actividad física del GPAQ. Se estudió el nivel de actividad física en una muestra de 54 adultos mayores, valorando la frecuencia en días, duración en minutos en los últimos 7 días y la intensidad de la actividad física expresando los resultados en METs$\mathrm{min} / \mathrm{semana}$. Arrojando los siguientes resultados con un $68 \%$ en nivel alto $24.1 \%$ en nivel medio y $11.1 \%$ en nivel bajo, distante de lo reportado por De Chimbo Yunga y cols., en el trabajo titulado "Nivel de actividad física medida a través del cuestionario internacional de actividad física, en los adultos mayores de las parroquias urbanas de Cuenca - Ecuador, 2015; donde los resultados fueron un $44.96 \%$ en nivel alto, $37.21 \%$ en nivel medio y un $17.83 \%$ en nivel bajo arrojados del instrumento de evaluación de IPAQ versión corta, ${ }^{(10)}$ la diferencia a pesar de que el rango de edad fue de 61 a 91 años de edad, mientras en comparación con el trabajo citado fue de 65 a 74 años, con una muestra de 387 adultos mayores.

En cuanto a los factores personales de los adultos mayores de La Misión en el género el sexo masculino $40.7 \%$ y femenino es el $59.3 \%$; en el trabajo presentado por De Chimbo y cols., mostró $36.6 \%$ en sexo masculino y $63.6 \%$ en femenino, en cuanto al estado civil presentó un $12.1 \%$ soltero, casado un $55.8 \%$, en divorciado un $10.1 \%$, unión libre un $1.1 \%$ y viudo un $20.9 \%$, ${ }^{(10)}$ en el estudio presente se dio un porcentaje similar en el estado civil soltero un $11.1 \%$, casado el $27.8 \%$, divorciado con $3.7 \%$, en unión libre $25.9 \%$ y viudo con $31.5 \%$.

En el trabajo De Chimbo Yunga y cols., en cuanto a la escolaridad el $33.3 \%$ tenía primaria incompleta, un $35.4 \%$ primaria completa, en secundaria solo el $9.1 \%$, fue incompleta y un $14.2 \%$ completa, en educación superior un $2.1 \%$ fue incompleta y un $5.9 \%$ completo, de acuerdo a la ocupación laboral el $26.1 \%$ es activo y un $73.9 \%$ es inactivo ${ }^{(10)}$ a diferencia a nuestra muestra población se tiene un nivel bajo en estudios contando con el $25.9 \%$ sin estudio, el $59.3 \%$ con solo la primaria y $14.8 \%$ con secundaria, en la ocupación destaca el $59.3 \%$ en el hogar, un $5.6 \%$ un trabajo laboral y un $35.2 \%$ trabaja en el campo.

\section{Conclusiones.}

En la población estudiada predomina un nivel alto de actividad física influyendo los factores personales como lo son género y la religión, haciendo notar la diferenciade actividades que realizan cada uno de ellas.

La autoestima del adulto mayor es elevada, prevalece la unión libre, sin estudios, las mujeres se ocupan al hogar mientras los hombres al campo y sus ingresos son obtenidos por parte de apoyo gubernamental. Estos factores personales no influyen en la actividad física del adulto mayor de La Misión Hidalgo.

Es importante indagar la influencia de los demás factores personales que se asocien en los niveles bajos de actividad física, y así contribuir a promocionare incentivar para la mejora de estos. Para así poner especial atención a estos espacios en lugares rurales para una vida más activa y productiva en beneficio de los adultos mayores para que en el nivel no disminuya. 


\section{Referencias}

[1] Organización Mundial de la Salud. Physical Activity [Internet] OMS; 2018. [consultado 2020 oct 28] Disponible en: http://www.who.int/news-room/fact-sheets/detail/physical-activity.

[2] Hernández-Soto OJ. Actividad física en adultos de edad avanzada: la alternativa promotora de salud. Umbral [Internet]. 2015 [consultado 2020 oct 28];10:76-107. Disponible en: http://umbral.uprrp.edu/revista/n10-vejez/actividad-fisica-en-adultosde-edad-avanzada-la-alternativa-promotora-de-salud/

[3] Alvarado-García, AM, Salazar-Maya ÁM. Análisis del concepto de envejecimiento. Gerokomos [Internet] 2014 [consultado 2020 oct 28];25(6):57-62. Disponible en: http://scielo.isciii.es/pdf/geroko/v25n2/revision1.pdf

[4] Maldonado-Muñiz, G., Arana-Gómez, B., Cárdenas-Becerril, L. Efecto del cuidado profesional de enfermería en el estilo de vida del adulto mayor que vive con diabetes [Internet]. Toluca, Estado de México: Universidad Autónoma del Estado de México; 2018 [consultado 2020 oct 28]. Disponible en: http://ri.uaemex.mx/bitstream/handle/20.500.11799/99141/TESIS\%20 DOCTORADO\%20GMM.\%20DICIEMBRE\%202018.pdf?sequence= 1 \&isAllowed $=\mathrm{y}$

[5] Organización Mundial de la Salud. Actividad física [Internet] OMS; 2019. [consultado 2020 oct 28] Disponible en: https://www.who.int/dietphysicalactivity/pa/es/

[6] Organización Mundial de la Salud. La actividad física en los adultos mayores [Internet] OMS; 2019. [consulta do 2020 oct 28] Disponible en: https://www.who.int/dietphysicalactivity/factsheet_olderadults/es/

[7] Pender JN. Modelo de promoción de la salud. En: Marriner A, Raile M, editoras. Modelos y teorías en enfermería. Madrid: Elsevier; 2011. p. $132-157$

[8] Jurado-Cárdenas D, Jurado-Cárdenas S, López-Villa gran K, QuerevalúGuzmán, B. Validez de la Escala de Autoestima de Rosenberg en universitarios de la Ciudad de México. Revista Lationamericana de Medicina Conductual [Internet] 2014 [consultado 2020 oct 21]; 5(1): 18-22. Disponible https://www.cop.es/colegiados/pv00520/escala\%20rosenberg.pdf

[9] Organización Mundial de la Salud. Vigilancia global de la actividad física [Internet] OMS; 2017. [consultado 2020 oct 25] Disponible en: http://www.who.int/chp/steps/GPAQ/es/

[10] Chimbo-Yunga J, Chuchuca-Cajamarca A, Encalada-Torres L, Wong S. Nivel de actividad física medida a través del cuestionario internacional de actividad física, en adultos mayores de las parroquias urbanas de Cuenca-Ecua dor. [Internet] 2016 [consulta do $2020 \mathrm{feb} 10$ ]; 34(2):51-56. Disponible en: https://publicaciones.ucuenca.edu.ec/ojs/index.php/medicina/article/vie w/990/878 